\title{
Laser-assisted decay spectroscopy and mass spectrometry of ${ }^{178} \mathrm{Au}$
}

J. G. Cubiss $\odot,{ }^{1, *}$ A. N. Andreyev $\odot,{ }^{1,2}$ A. E. Barzakh, ${ }^{3}$ V. Manea $\odot,{ }^{4, \dagger}$ M. Al Monthery, ${ }^{1}$ N. A. Althubiti,${ }^{5,6}$ B. Andel $\odot,{ }^{7,8}$ S. Antalic $\odot,{ }^{8}$ D. Atanasov, ${ }^{4,9}$ K. Blaum, ${ }^{4}$ T. E. Cocolios $\odot,{ }^{5,7}$ T. Day Goodacre, ${ }^{5,9, *}$ A. de Roubin, ${ }^{4,}{ }^{8}$ G. J. Farooq-Smith, ${ }^{5,7}$ D. V. Fedorov, ${ }^{3}$ V. N. Fedosseev $\odot,{ }^{9}$ D. A. Fink,,${ }^{9,4}$ L. P. Gaffney $\odot,{ }^{7,9, \|}$ L. Ghys, ${ }^{7, \pi}$ R. D. Harding $\odot,{ }^{1,9}$ F. Herfurth, ${ }^{10}$ M. Huyse, ${ }^{7}$ N. Imai, ${ }^{11}$ D. T. Joss, ${ }^{12}$ S. Kreim,,${ }^{9,4}$ D. Lunney, ${ }^{13,}{ }^{\dagger}$ K. M. Lynch,${ }^{5,9}$ B. A. Marsh, ${ }^{9}$ Y. Martinez Palenzuela, ${ }^{7,9}$ P. L. Molkanov, ${ }^{3}$ D. Neidherr, ${ }^{10}$ G. G. O’Neill, ${ }^{12}$ R. D. Page, ${ }^{12}$ M. Rosenbusch, ${ }^{14}$ R. E. Rossel,${ }^{9,15}$ S. Rothe, ${ }^{9,15}$ L. Schweikhard, ${ }^{14}$ M. D. Seliverstov,${ }^{3}$ S. Sels $\odot,{ }^{7}$ A. Stott, ${ }^{1}$ C. Van Beveren, ${ }^{7}$ P. Van Duppen $\odot,{ }^{7}$ E. Verstraelen $\odot,{ }^{7}$ A. Welker, ${ }^{9,16}$ F. Wienholtz, ${ }^{9,14, \#}$ R. N. Wolf,,${ }^{414, * *}$ and K. Zuber ${ }^{16}$

${ }^{1}$ Department of Physics, University of York, York YO10 5DD, United Kingdom

${ }^{2}$ Advanced Science Research Center (ASRC), Japan Atomic Energy Agency, Tokai-mura, Japan

${ }^{3}$ Petersburg Nuclear Physics Institue, NRC Kurchatov Institute, 188300 Gatchina, Russia

${ }^{4}$ Max-Planck-Institut für Kernphysik, Saupfercheckweg 1, 69117 Heidelberg, Germany

${ }^{5}$ The University of Manchester, School of Physics and Astronomy, Oxford Road, Manchester M13 9PL, United Kingdom

${ }^{6}$ Physics Department, Faculty of Science, Jouf University, Aljouf, Saudi Arabia

${ }^{7}$ KU Leuven, Instituut voor Kern- en Stralingsfysica, 3001 Leuven, Belgium

${ }^{8}$ Department of Nuclear Physics and Biophysics, Comenius University in Bratislava, 84248 Bratislava, Slovakia

${ }^{9}$ CERN, 1211, Geneva 23, Switzerland

${ }^{10}$ GSI Helmholtzzentrum für Schwerionenforschung GmbH, 64291 Darmstadt, Germany

${ }^{11}$ Center for Nuclear Study (CNS), Graduate School of Science, The University of Tokyo, Japan

${ }^{12}$ Department of Physics, University of Liverpool, Liverpool L69 7ZE, United Kingdom

${ }^{13}$ CSNSM-CNRS, Université de Paris Sud, 91400 Orsay, France

${ }^{14}$ Institut für Physik, Universität Greifswald, 17487 Greifswald, Germany

${ }^{15}$ Institut für Physik, Johannes Gutenberg-Universität Mainz, 55128 Mainz, Germany

${ }^{16}$ Institut für Kern- und Teilchenphysik, Technische Universität Dresden, 01069 Dresden, Germany

(Received 27 July 2020; accepted 23 September 2020; published 30 October 2020; corrected 14 October 2021)

\begin{abstract}
A comprehensive study of the isotope ${ }^{178} \mathrm{Au}$ has been made at the CERN-ISOLDE facility, using resonance laser ionization. Two long-lived states in ${ }^{178} \mathrm{Au}$ were identified-a low-spin ground state and a high-spin isomer-each of which were produced as pure beams. Using the ISOLTRAP precision Penning trap, the excitation energy of the isomeric state in ${ }^{178} \mathrm{Au}$ was determined to be $E^{*}=189(14) \mathrm{keV}$. The $\alpha$-decay fine structure patterns of the two states were studied using the Windmill decay station, providing information on the low-lying states in the daughter nucleus ${ }^{174} \mathrm{Ir}$. Nuclear spin assignments of $I\left({ }^{178} \mathrm{Au}^{g}\right)=(2,3)$ and $I\left({ }^{178} \mathrm{Au}^{m}\right)=(7,8)$ are made based on the observed $\beta$-decay feeding and hyperfine structure intensity patterns. These spin assignments are used for fitting the hyperfine structures of the two states from which values for the magnetic dipole moments are extracted. The extracted moments are compared with calculations using additivity relations to establish the most probable configurations for ${ }^{178} \mathrm{Au}^{g, m}$.
\end{abstract}

DOI: $10.1103 /$ PhysRevC.102.044332

*james.cubiss@york.ac.uk

†Present address: Université Paris-Saclay, CNRS/IN2P3, IJCLab, 91405 Orsay, France.

†Present address: TRIUMF, Vancouver BC V6T 2A3, Canada.

${ }^{\S}$ Present address: Centre d'Etudes Nucléaires de Bordeaux-Gradignan, 19 Chemin du Solarium, CS 10120, F-33175 Gradignan, France.

"Present address: Department of Physics, University of Liverpool, Liverpool L69 7ZE, United Kingdom.

IIPresent address: Belgian Nuclear Research Center SCK-CEN, Boeretang 200, B-2400 Mol, Belgium.

\#Present address: Institut für Kernphysik, Technische Universität Darmstadt, 64289 Darmstadt, Germany.

${ }^{* * *}$ Present address: ARC Centre of Excellence for Engineered Quantum Systems, The University of Sydney, NSW 2006, Australia.

Published by the American Physical Society under the terms of the Creative Commons Attribution 4.0 International license. Further distribution of this work must maintain attribution to the author(s) and the published article's title, journal citation, and DOI. 


\section{INTRODUCTION}

The application of lasers and traps at ISOL (isotope seprator online) facilities has substantially advanced the range and depth of spectroscopic and spectrometric studies of nuclei due to the high selectivity of the techniques involved [1-6]. In particular, it is now possible to produce and study isomerically pure beams of selected nuclear states by exploiting differences in their respective hyperfine structures (hfs) [7].

Neutron-deficient gold $(Z=79)$ isotopes, being three protons below the $Z=82$ shell closure, are known to exhibit shape coexistence. Specifically, approaching the $N=$ 104 neutron midshell, deformed configurations involving the $\pi i_{13 / 2}, \pi h_{9 / 2}$ and $\pi f_{7 / 2}$, intruder states have been observed at low energy, alongside near spherical $\pi s_{1 / 2}, \pi d_{3 / 2}$, and $\pi h_{11 / 2}$ configurations [8-10]. As early as the 1980s, charge radii studies demonstrated an abrupt jump from a near-spherical ground state in ${ }^{187} \mathrm{Au}(N=108)$ to a strongly deformed one in ${ }^{183-186} \mathrm{Au}(N=104-107)$ [11,12]. Rotational bands built on top of intruder states have been observed in a number of odd- $A$ isotopes [13-16], with bandhead energies showing the typical parabolic behavior as a function of neutron number (see Fig. 1 in Ref. [8]).

Recently, an extended campaign to measure the isotope shifts, hfs, and decay properties of gold isotopes beyond the neutron midshell has been performed at the CERN-ISOLDE facility, with specific emphasis on the neutron-deficient isotopes. The combination of laser-, decay-, and massspectroscopy techniques provides unprecedented purification that allows fine probing of the physics occurring in this region. Partial results from this campaign were published for the isotopes ${ }^{177,179} \mathrm{Au}^{m}$ [17], ${ }^{180,182} \mathrm{Au}^{m}$ [18], ${ }^{187} \mathrm{Au}^{m}$ [19], and 177,191,193,195 $\mathrm{Au}^{m}$ [20].

In this work we report on a study of the nucleus ${ }^{178} \mathrm{Au}(N=$ 99), which lies near the predicted end of strongly deformed ground states in gold nuclei [21]. Therefore, the properties of this nucleus may be important for understanding shape evolution and shape coexistence in this region of the nuclear chart.

The earliest study of ${ }^{178} \mathrm{Au}$ by Siivola using a He-jet technique [22] identified an $\alpha$ decay from ${ }^{178} \mathrm{Au}$ with an energy of $5920(10) \mathrm{keV}$ and a half-life of $T_{1 / 2}=2.6(5) \mathrm{s}$. A subsequent investigation by Keller et al. at the velocity filter SHIP [23] observed additional $\alpha$ decays with energies of 5850(20) and $5980 \mathrm{keV}^{1}$ and placed a lower limit on the $\alpha$-decay branching ratio of $b_{\alpha}\left({ }^{178} \mathrm{Au}\right) \geqslant 40 \%$. All three decays were attributed to a single state in ${ }^{178} \mathrm{Au}$. In these two earlier studies, complex $\alpha$-decay spectra had to be analyzed due to admixtures from different competing evaporation channels (e.g., $x n$, pxn, and $\alpha x n)$ of the complete fusion reactions used in both studies. However, a later $\beta^{+} / \mathrm{EC}$ (electron capture) study of ${ }^{178} \mathrm{Au}$ [24] suggested that, based on the observed feeding pattern to levels in the daughter nucleus, ${ }^{178} \mathrm{Pt}$, there may be two $\beta$-decaying states in ${ }^{178} \mathrm{Au}$ with spins of $\approx 2$ and $\approx 8$.

\footnotetext{
${ }^{1}$ No uncertainty is given in the original publication or the evaluated data for the $5980-\mathrm{keV}$ decay.
}

Also pertinent to the present work is information on the low-lying structure of the $\alpha$-decay daughter nucleus, ${ }^{174} \mathrm{Ir}$, which primarily comes from an $\alpha$ and $\beta$ decay study that also used the He-jet method [25]. Here, two $\alpha$-decaying states were identified: an $I^{\pi}=\left(3^{+}\right)$ground state and an $I^{\pi}=\left(7^{+}\right)$ isomeric state at an excitation energy of 193(11) keV. The spin and parity assignments for these states were based on the observed $\beta$-decay feeding patterns to levels in ${ }^{174} \mathrm{Os}$.

In the present work we exploited the Resonant Ionization Laser Ion Source (RILIS) [1,26] and the ISOLDE General Purpose Separator (GPS) [27]. With these it was possible to create uniquely clean beams of ${ }^{178} \mathrm{Au}^{g, m}$ that were unattainable in the previous studies.

\section{EXPERIMENTAL SETUP}

Nuclei of ${ }^{178} \mathrm{Au}$ were produced in spallation reactions induced by impinging a beam of protons with an energy of $1.4 \mathrm{GeV}$ and a maximum current of $2.1 \mu \mathrm{A}$ onto a $50 \mathrm{~g} \mathrm{~cm}^{-2}$ $\mathrm{UC}_{x}$ target. The CERN PS Booster delivered the proton beam in a repeated sequence comprising a so-called "supercycle", which typically consisted of 35-40, 2- $\mu$ s-long proton pulses, separated by $1.2 \mathrm{~s}$.

After proton impact, reaction products diffused through the target material and effused towards a hot cavity ion source [28], kept at a temperature of $\approx 2000-2200{ }^{\circ} \mathrm{C}$. Inside the cavity, gold isotopes were selectively ionized using three laser beams wavelength tuned to the atomic transitions, as shown in Fig. 1(a). A combination of a narrow-bandwidth Ti:sapphire (Ti:Sa) and two broad-bandwidth dye lasers (BBDL) was operated synchronously at a pulse repetition rate of $10 \mathrm{kHz}$. Ultraviolet radiation required for the first and second steps of the atomic excitation was produced by frequency tripling and doubling of the emission produced by Ti:Sa and BBDL, respectively.

The ions were then extracted by a $30 \mathrm{kV}$ potential and further separated according to their mass-to-charge ratio by the GPS. Following this, the ions were transported to either the ISOLTRAP mass spectrometer [29,30], discussed in Sec. III B, or to the Windmill (WM) system [31,32] for the decay measurements described in Sec. III C 1.

At the WM, the ion beam entered through the central hole of an annular silicon detector (Si1), and was implanted into one of ten, $20-\mu \mathrm{g} \mathrm{cm}^{-2}$-thick carbon foils mounted on a rotatable wheel (see Fig. 2 in Ref. [32]). A second silicon detector ( $\mathrm{Si} 2)$ was positioned a few millimeters behind the foil being irradiated. Together, $\mathrm{Si} 1+\mathrm{Si} 2$ were used to measure the short-lived $\alpha$-decay activity at the implantation site. After each supercycle, the wheel of the WM was rotated (within $\approx 0.8 \mathrm{~s}$ ), positioning a fresh foil at the implantation site. The energy calibration of the detectors was performed using the $\alpha$ decay of ${ }^{178} \mathrm{Pt}\left(E_{\alpha}=5446(3) \mathrm{keV}\right.$ [33]), which was produced by the $\beta^{+} / \mathrm{EC}$ decay of ${ }^{178} \mathrm{Au}$, and of other $\alpha$-decaying nuclides studied in the same experiment at different mass settings of ISOLDE.

A single crystal low-energy germanium (LEGe) detector was placed outside the WM chamber, directly behind Si2. The typical energy resolution (full width at half maximum) and detection efficiency of the LEGe for $121.8-\mathrm{keV} \gamma$ radiation 


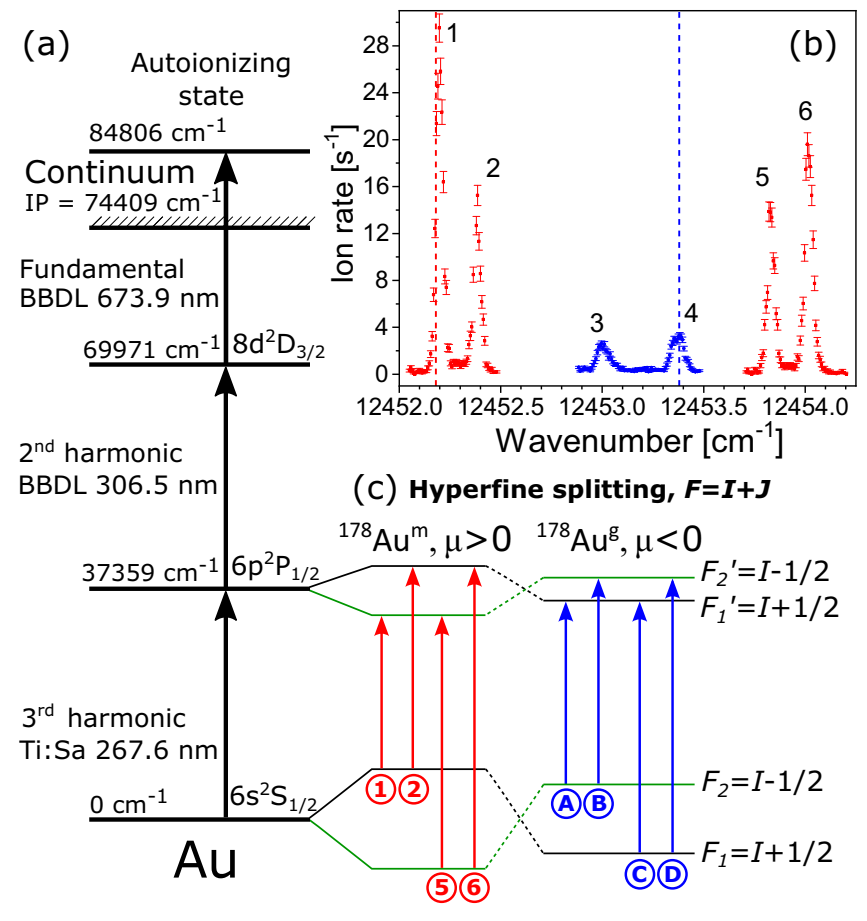

FIG. 1. (a) The three-step laser-ionization scheme used to produce the gold ions (see text for details); (b) the hyperfine structure spectrum of ${ }^{178} \mathrm{Au}$ (rate of photo-ions measured by the MR-ToF MS as a function of the wave number of the first-step laser, before tripling, used in the ionization process). Peaks 1, 2, 5, and 6 (red data points) belong to ${ }^{178} \mathrm{Au}^{m}$ and peaks 3 and 4 (blue data points) to ${ }^{178} \mathrm{Au}^{g}$; (c) the hyperfine levels (not to scale) for ${ }^{178} \mathrm{Au}^{m}(\mu>0)$ and ${ }^{178} \mathrm{Au}^{g}(\mu<0)$. The relative spacing and ordering of the hfs levels for ${ }^{178} \mathrm{Au}^{g, m}$ indicate the difference in the magnitude and sign of their respective magnetic dipole moments (see Sec. IIIE). The transitions between hyperfine states for ${ }^{178} \mathrm{Au}^{g}$ are indicated by the blue arrows. Due to Doppler broadening, transitions A and B overlap and their combined intensity is shown as peak 3 in panel (b); similarly transitions $\mathrm{C}$ and $\mathrm{D}$ overlap and combine to make peak 4. For ${ }^{178} \mathrm{Au}^{m}$, all four transitions are well separated; the transition label numbers correspond to the peak numbers in panel (b).

were $1.1 \mathrm{keV}$ and $7.7 \%$, respectively. Energy and efficiency calibrations were made using calibration sources of ${ }^{137} \mathrm{Cs}$, ${ }^{133} \mathrm{Ba},{ }^{60} \mathrm{Co}$, and ${ }^{152} \mathrm{Eu}$.

\section{RESULTS}

\section{A. Hyperfine structure measurements}

The initial identification of two long-lived states in ${ }^{178} \mathrm{Au}$ was based on hyperfine splitting measurements performed by counting the number of $\alpha$ decays with the WM decay station or the number of photo-ions using a multireflection time-of-flight mass spectrometer (MR-ToF MS) [30]. Here, the frequency of the first-step laser used for the 267.6-nm transition was scanned in order to measure the hfs of the $6 s^{2} S_{1 / 2}$ and $6 p^{2} P_{1 / 2}$ levels. The fundamental frequency output of the Ti:Sa laser was measured using a laser wavelength meter (model WS7 by HighFinesse/Angstrom). In total, three hfs scans were performed, one using the WM and two using

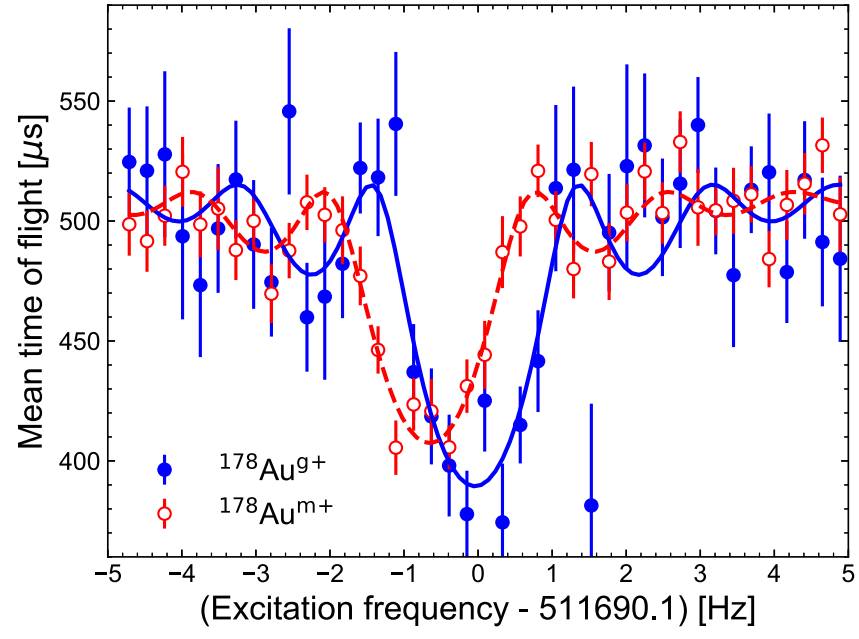

FIG. 2. Time-of-flight ion-cyclotron resonances of the two longlived states in ${ }^{178} \mathrm{Au}$ measured with ISOLTRAP. The blue, filled symbols are the data points for ${ }^{178} \mathrm{Au}^{g}$, and the red, hollow symbols are for ${ }^{178} \mathrm{Au}^{m}$. The fits of the theoretical line shapes [35] are also shown by the solid blue line for ${ }^{178} \mathrm{Au}^{g}$ and by the dashed red line for ${ }^{178} \mathrm{Au}^{m}$. The data were collected separately using the different RILIS settings (see text for details).

the MR-ToF MS. Details of the scanning procedures are given in Ref. [34]. An example of the hfs spectrum measured by the MR-ToF MS is shown in Fig. 1(b), in which six peaks can be seen.

The decay data collected by the WM during the hfs scan indicated that peaks 1, 2, 5, and 6 in Fig. 1(b) belong to the same nuclear state, which was identified as the isomer $\left({ }^{178} \mathrm{Au}^{m}\right)$ during the dedicated mass measurements discussed in Sec. III B. Similarly, it was found that peaks 3 and 4 in Fig. 1(b) belong to the ground state, ${ }^{178} \mathrm{Au}^{g}$. However, the relatively small magnetic dipole moment of ${ }^{178} \mathrm{Au}^{g}$ combined with the Doppler broadening $(\approx 2.9 \mathrm{GHz})$ of the $267.6 \mathrm{~nm}$ transition due to the velocity spread of atoms in the hot ion source meant that it was not possible to resolve the hfs of the upper atomic level. Hence, only two peaks are seen in Fig. 1(b) belonging to ${ }^{178} \mathrm{Au}^{g}$, whereby peak 3 is the sum of transitions $F_{2} \rightarrow F_{1}^{\prime}, F_{2}^{\prime}$ labeled $\mathrm{A}$ and $\mathrm{B}$ in Fig 1(c) and similarly, peak 4 is the combination of transitions $\mathrm{C}$ and $\mathrm{D}$.

The dedicated mass- and decay-spectroscopy measurements described in the following sections were taken with the laser wave number set to two fixed values in order to produce isomerically pure ion beams. Settings of $12453.38 \mathrm{~cm}^{-1}$ and $12452.18 \mathrm{~cm}^{-1}$ [indicated by the dashed vertical lines in Fig. 1(b)] were used for ${ }^{178} \mathrm{Au}^{g}$ and ${ }^{178} \mathrm{Au}^{m}$, respectively. At these laser settings, the ion beam intensities after the GPS separator were $\approx 3 \times 10^{3}$ and $4 \times 10^{2}$ ions $\mu \mathrm{C}^{-1}$ for ${ }^{178} \mathrm{Au}^{g, m}$, respectively.

\section{B. Direct determination of the isomer excitation energy}

For the mass measurements, the isomerically pure beams were transported to the Penning-trap system of ISOLTRAP [29]. The MR-ToF MS was used in this case to purify the ${ }^{178} \mathrm{Au}^{+}$beam from isobaric contamination, consisting of 
surface-ionized molecules of lighter elements. At peak laserionization efficiency the contaminants were $\approx 4$ times more intense than ${ }^{178} \mathrm{Au}^{m}$, and $\approx 20$ times more than ${ }^{178} \mathrm{Au}^{g}$. A final bunching and cooling of the ions was performed in the preparation Penning trap, after which they were transferred to the precision Penning trap where their cyclotron frequency was determined using the time-of-flight ion-cyclotron resonance (ToF-ICR) technique [35]. The Penning-trap magnetic field was calibrated by the measured cyclotron frequency of ${ }^{133} \mathrm{Cs}^{+}$ ions from an offline ion source, which have a well-known mass. Finally, the cyclotron-frequency ratio $r$ between the reference ion ref and the ion of interest $x, r=v_{c, \text { ref }} / v_{c, x}=$ $m_{\text {ion, } x} / m_{\text {ion,ref }}$, was measured (an expression involving ion masses), from which one can extract the atomic mass of ${ }^{178} \mathrm{Au}$ as

$$
m_{x}=r\left(m_{\mathrm{ref}}-m_{e}\right)+m_{e},
$$

where $m_{e}$ is the electron mass and $m_{\text {ref }}, m_{x}$ are the atomic masses of the reference nuclide and the nuclide of interest, respectively (both as neutral atoms).

The ToF-ICR measurements performed on the pure ground-state and isomeric-state samples are shown in Fig. 2. For details on the analysis technique, see Ref. [37]. The resulting cyclotron-frequency ratios are presented in Table I. The uncertainty of the ground-state mass was significantly improved, and the excitation energy of the newly established isomeric state was determined for the first time to be 189(14) keV.

\section{Decay spectroscopy \\ 1. $\alpha$-decay spectra}

Figures 3(a) and 3(b) show the combined singles $\alpha$-decay spectra measured in $\mathrm{Si} 1$ and $\mathrm{Si} 2$ for ${ }^{178} \mathrm{Au}^{g}$ and ${ }^{178} \mathrm{Au}^{m}$, respectively. The peaks at 5291(10) and 5446(5) keV are the $\alpha$ decays of ${ }^{178} \mathrm{Pt}$.

Three peaks which we attribute to the decay of ${ }^{178} \mathrm{Au}^{g}$ are seen in Fig. 3(a) at energies of $E_{\alpha}=5750(10), 5840(10)$, and $5922(5) \mathrm{keV}$, along with a weaker fourth peak at 5811(10) keV that was identified in the analysis of the $\alpha-\gamma$ coincidence data discussed in the following section. In contrast to this, five $\alpha$ decay peaks are observed in Fig. 3(b) for ${ }^{178} \mathrm{Au}^{m}$, at 5521(10), 5571(10), 5839(10), 5925(7), and 5977(10) keV.

The intensities $\left(I_{\alpha}\right)$ of the different fine structure (f.s.) decays were deduced by fitting the spectra shown in Figs. 3(a)

TABLE I. Frequency ratios with respect to ${ }^{133} \mathrm{Cs}^{+}$and mass excess (ME) values of ${ }^{178} \mathrm{Au}^{g, m}$ measured in this work. The wave number of the first excitation step of RILIS used to produce the two states, as well as the mass-excess value of ${ }^{178} \mathrm{Au}^{g}$ from AME 2012 [36] are also presented.

\begin{tabular}{lcc}
\hline \hline State & ${ }^{178} \mathrm{Au}^{g}$ & ${ }^{178} \mathrm{Au}^{m}$ \\
\hline RILIS setting $\left(\mathrm{cm}^{-1}\right)$ & 12453.38 & 12452.18 \\
$r=v_{c, \text { ref }} / v_{c}$ & $1.3391191998(798)$ & $1.3391207228(827)$ \\
$\mathrm{ME}_{\text {ISOLTRAP }}\left(\mathrm{keV} / c^{2}\right)$ & $-22303.3(99)$ & $-22114.7(102)$ \\
$\mathrm{ME}_{\text {AME 2012 }}\left(\mathrm{keV} / c^{2}\right)$ & $-22330(60)$ & \\
\hline \hline
\end{tabular}

and 3(b) with Crystal Ball functions [38]. In the case of ${ }^{178} \mathrm{Au}^{m}$, a significant component of the 5977-keV decay peak is due to $\alpha$-conversion electron $\left(\alpha-e^{-}\right)$summing with the $5925-\mathrm{keV} \alpha$ decay which had to be accounted for (see discussion in Sec. III C 4).

\section{2. $\alpha-\gamma$ coincidences for ${ }^{178} \mathrm{Au}^{g}$}

Figure 3(c) shows the prompt $(\Delta T \leqslant 210 \mathrm{~ns}) \alpha-\gamma$ coincidence data between $\mathrm{Si} 1+\mathrm{Si} 2$ and the LEGe detector for the singles events in Fig. 3(a). Four groups of strong $\alpha-\gamma$ coincidence events can be identified at $5840(10)-82.8(3) \mathrm{keV}$, 5840(10)-90.0(3) keV, 5811(10)-115.7(3) keV, and 5750(15)$174.8(5) \mathrm{keV}$. The 5750- and 5811-keV decays are also seen in coincidence with a weak 98.3(5)-keV transition. Due to the high intensity of the $170 \mathrm{keV} 2_{1}^{+} \rightarrow 0_{1}^{+}$in ${ }^{178} \mathrm{Pt}$ (see Sec. III D 1), a small number of time-random 5922-170 keV and 5922-Pt $K_{\alpha, \beta}$ X rays are observed in Fig. 3(c). The projection on the $E_{\gamma}$ axis for the $5720 \leqslant E_{\alpha} \leqslant 5880 \mathrm{keV}$ region is shown in Fig. 3(e). It shows a strong presence of iridium $K_{\alpha, \beta}$ $\mathrm{x}$ rays at 63.3(3), 64.8(3), 73.5(3), and 75.5(3) keV, in good agreement with tabulated values. Projections on the $E_{\alpha}$ axis for the observed $\alpha-\gamma$ coincidences are shown in Fig. 4.

The decay scheme for ${ }^{178} \mathrm{Au}^{g}$ shown in Fig. 5 is built by comparing the $Q_{\alpha, t o t}=Q_{\alpha}+E_{\gamma}$ values of the $\alpha-\gamma$ groups to a reference $Q_{\alpha, g}$ value for the $5922-\mathrm{keV}{ }^{178} \mathrm{Au}$ ground-state decay. This decay is assigned as directly feeding to ${ }^{174} \mathrm{Ir}^{g}$, such that $Q_{\alpha, g}=6058(5) \mathrm{keV}$.

The $Q_{\alpha, t o t}=6064(10), 6060(10)$, and $6057(15) \mathrm{keV}$ values for the 5840-90.0, 5811-115.7, and 5750-174.8 keV groups are in good agreement with $Q_{\alpha, g}$. This establishes three levels at excitation energies of 90.0(3), 115.7(3), and 174.8(5) $\mathrm{keV}$ in ${ }^{174} \mathrm{Ir}^{g}$.

The 5840-82.8 keV group indicates that the 90.0-keV level also deexcites by an $82.8-\mathrm{keV}$ transition. As this transition is prompt, we assign it as feeding to a state at 7.2(4) $\mathrm{keV}$ which deexcites by an unobserved transition.

The 98.3-keV transition in coincidence with the 5811and 5750-keV decays [see Fig. 4(c)] has low statistics and does not match the energy differences between the established states. Therefore, we cannot place it in the decay scheme with certainty with our current data.

All the $\gamma$ rays in coincidence with the ${ }^{178} \mathrm{Au}^{g} \alpha$ decays are observed to be prompt, which limits their multipolarities to $\Delta L \leqslant 2$. For the 90.0 - and $82.8-\mathrm{keV}$ decays, the multipolarities are deduced by comparing the number of counts in the $5840-82.8 \mathrm{keV}$ and 5840-90.0 keV coincidence groups with the number of iridium $K_{\alpha, \beta}$ x rays in coincidence with 5840$\mathrm{keV} \alpha$ decays [see inset of Fig. 3(e)]. The large number of $K$ $\mathrm{x}$ rays can only be explained if both the $82.8-$ and $90.0-\mathrm{keV}$ $\gamma$ rays are $M 1$ transitions. This conclusion is consistent with the number of 5840-keV events shown in Fig. 3(a), relative to the observed number of $\alpha-\gamma$ coincidences. Based on these $M 1$ assignments, the relative intensities were calculated by using theoretical conversion coefficients taken from BRICC $\left(\alpha_{\text {tot, } t h}\right)$ [41] for pure $M 1$ transitions, yielding $I_{\text {rel }}(90.0)=81(2) \%$ and $I_{\text {rel }}(82.8)=19(2) \%$.

The 5811- and 5750-keV $\alpha$ decays are also in coincidence with iridium $K$ x rays. However, some of these counts 

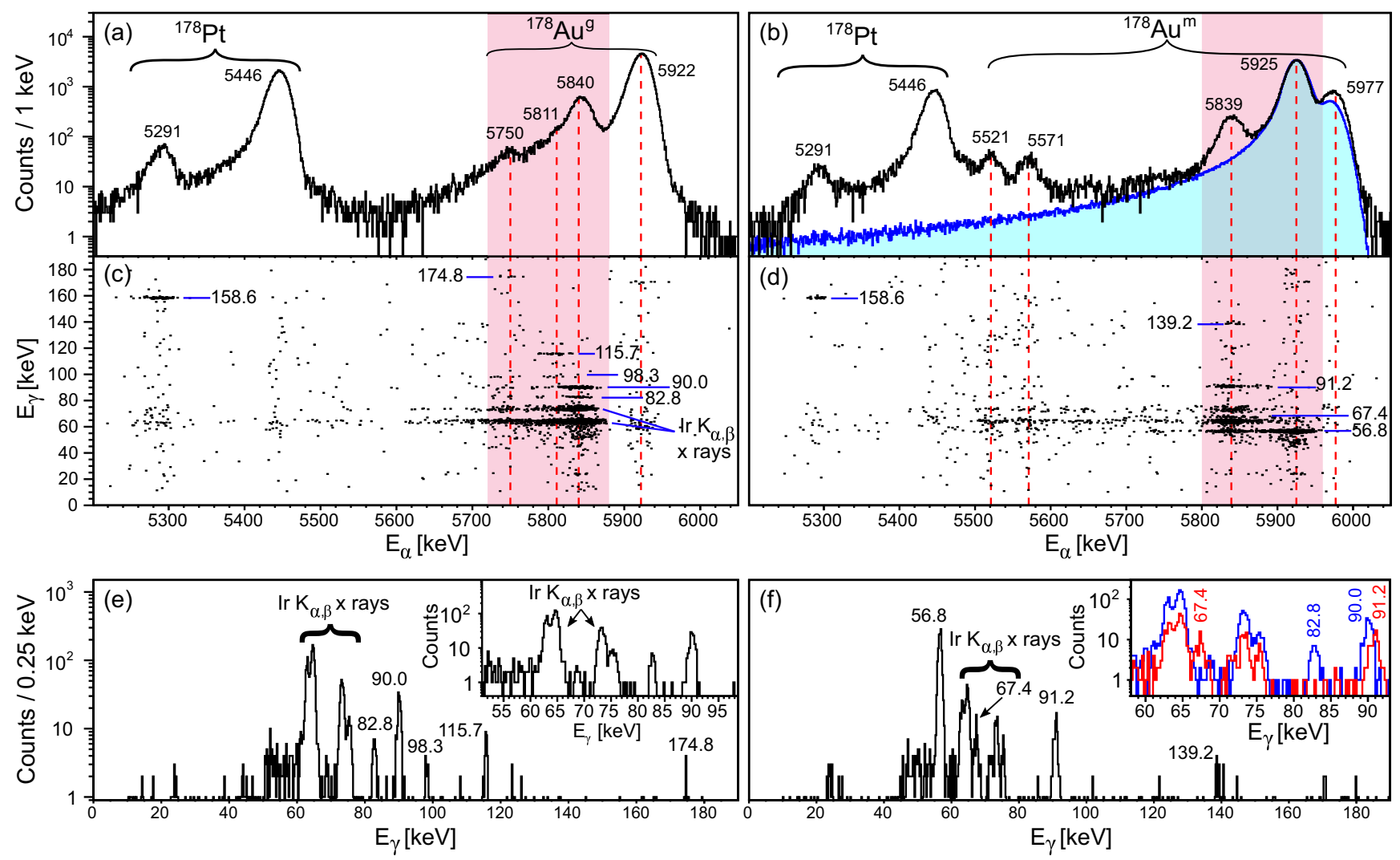

FIG. 3. Decay spectra for ${ }^{178} \mathrm{Au}^{g}$ (left) and for ${ }^{178} \mathrm{Au}^{m}$ (right). Panels (a) and (b): singles $\alpha$-decay spectra, measured in Si1 + Si2; the blue histogram in panel (b) shows the results from GEANT4 simulations of a single 5925-keV $\alpha$ decay followed by internal conversion instead for a 56.8-keV M1 transition. Panels (c) and (d): the $\alpha-\gamma$ coincidences measured within the time interval $\Delta T(\alpha-\gamma) \leqslant 210 \mathrm{~ns}$ for ${ }^{178} \mathrm{Au}^{g, m}$, respectively. The vertical dashed red lines indicate the $E_{\alpha}$ centroids of the $\alpha-\gamma$ coincidence groups. Panels (e) and (f): projections upon the $E_{\gamma}$ axis for the $E_{\alpha}=5720-5880$ and 5800-5960 keV regions of panel (c) and (d) (areas shaded in pink), respectively. The inset of panel (e) shows the projection on the $E_{\gamma}$ axis for the $E_{\alpha}=5820-5860 \mathrm{keV}$ region of panel (c). The inset of panel (f) shows the overlap of the spectra in panels (e) and (f) for the $E_{\gamma}=58-93 \mathrm{keV}$ region, shown in blue and red, respectively.

are due to tailing from the $5840-\mathrm{keV}$ peak. Furthermore, we cannot rule out unobserved $\gamma$-decay branches with large $K$-conversion coefficients competing with the 174.8- and $115.7-\mathrm{keV}$ transitions, so we can only limit their multipolarities to $\Delta L \leqslant 2$.

\section{3. $\alpha-\gamma$ coincidences for ${ }^{178} \mathrm{Au}^{m}$}

The prompt $\alpha-\gamma$ coincidence data between $\mathrm{Si} 1+\mathrm{Si} 2$ and the LEGe detector for the singles events in Fig. 3(b) are shown in Fig. 3(d), and their projections on the $E_{\gamma}$ axis for the $5800 \leqslant E_{\alpha} \leqslant 5960 \mathrm{keV}$ region are given in Fig. 3(f). Five sets of $\alpha-\gamma$ coincidences are seen with the $5839-\mathrm{keV}$ decay in coincidence with 56.8(3)-, 67.4(3)-, 91.2(3)-, and 139.2(3)-keV $\gamma$ transitions, and a 5925(7)-56.8(3) keV group (projections on the $E_{\alpha}$ axis for these $\alpha-\gamma$ groups are shown in Fig. 6). We note here that, apart from the iridium $K_{\alpha, \beta}$ x rays, none of the transitions observed in Fig. 3(f) have the same energy as those in Fig. 3(e), indicating that there is no internal transition between ${ }^{178} \mathrm{Au}^{g, m}$.

Using the same approach as for ${ }^{178} \mathrm{Au}^{g}$, we define a reference full-energy value for ${ }^{178} \mathrm{Au}^{m}$ using the strongest 5925-56.8 keV group: $Q_{\alpha, m}=6118(7) \mathrm{keV}$. This value is in good agreement with $Q_{\alpha}=6114(10) \mathrm{keV}$ for the highest energy 5977-keV $\alpha$ line which we assign as feeding directly to the isomeric state in ${ }^{174} \mathrm{Ir}$.

The excitation energy of ${ }^{174} \mathrm{Ir}^{m}$, denoted $\Delta$, is determined using $Q_{\alpha, g}, Q_{\alpha, m}$ and the excitation energy of ${ }^{178} \mathrm{Au}^{m}$ deduced in Sec. III B, giving $\Delta=129(17) \mathrm{keV}$. However, for the convenience of presentation we will build the level scheme for ${ }^{174} \mathrm{Ir}^{m}$ relative to $\Delta$. Hence, we establish a level fed by the 5925-keV decay at $56.8 \mathrm{keV}$ above ${ }^{174} \mathrm{Ir}^{m}$, as shown in Fig. 5.

The $Q_{\alpha, t o t}=6121(10) \mathrm{keV}$ value for a possible 91.2-56.8 $\mathrm{keV}$ cascade following the $5839-\mathrm{keV}$ decay is in good agreement with $Q_{\alpha, m}$, establishing a level at 148.0(4) $\mathrm{keV}$ above ${ }^{174} \mathrm{Ir}^{m}$. The $5840-139.2 \mathrm{keV}$ group is missing $8.8(5) \mathrm{keV}$ relative to a $91.2-56.8 \mathrm{keV}$ cascade. Based on its observed promptness, we assign the $139.2-\mathrm{keV}$ transition as deexciting from the $148.0-\mathrm{keV}$ level, feeding to a state at $8.8(5) \mathrm{keV}$ above ${ }^{174} \operatorname{Ir}^{m}$.

The $Q_{\alpha, t o t}=6041(10) \mathrm{keV}$ for the $5839-67.4 \mathrm{keV}$ group is missing $\approx 77 \mathrm{keV}$ relative to $Q_{\alpha, m}$. The missing energy is slightly larger than the iridium $K_{\beta} \mathrm{x}$ ray energies $\left(E_{x}=\right.$ 73-76 keV). Thus, considering the statistics in the 5839$67.4 \mathrm{keV}$ group in the inset of Fig. 3(f) and in Fig. 6(b), a 5839-77 keV group should be observable. However, no such transition is seen. 


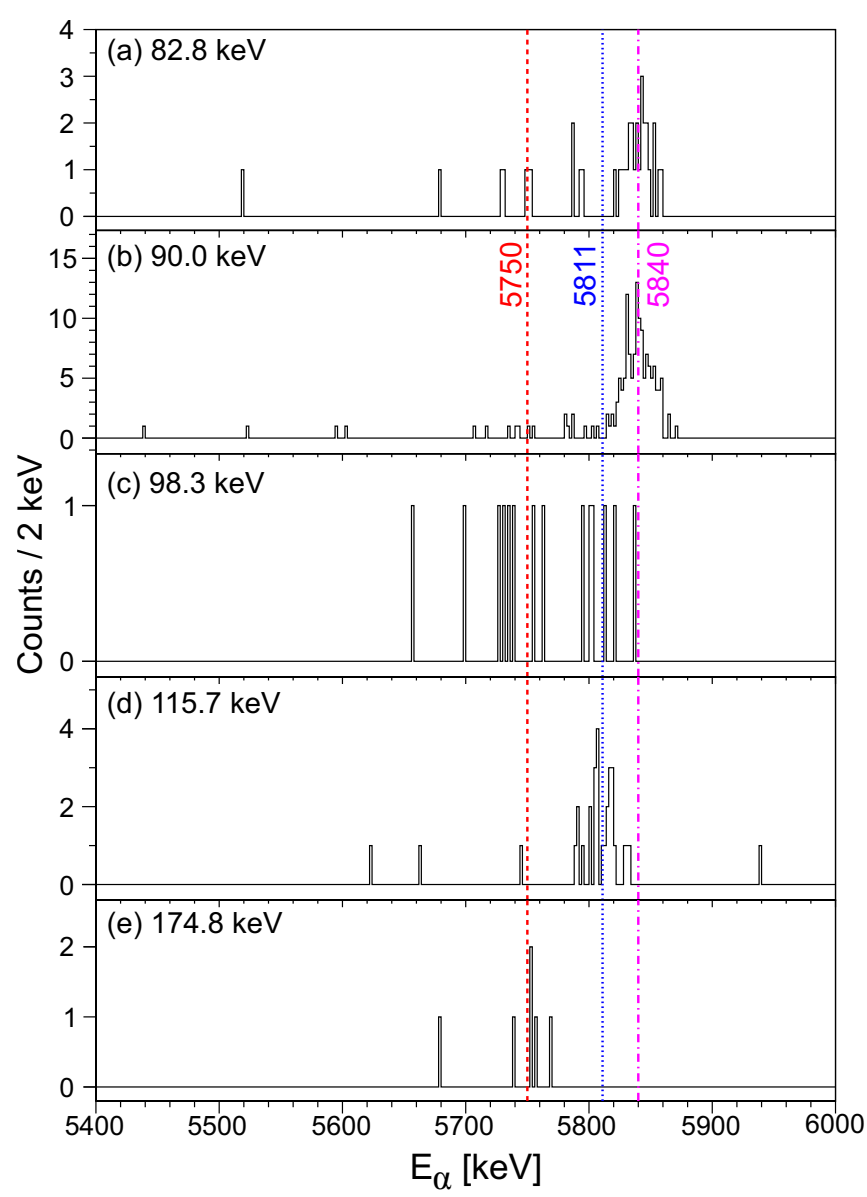

FIG. 4. Projections on the $E_{\alpha}$ axis for the $\alpha-\gamma$ groups of ${ }^{178} \mathrm{Au}^{g}$ data shown in Fig. 3(c), for the (a) 82.8-keV, (b) $90.0-\mathrm{keV}$, (c) 98.3$\mathrm{keV}$, (d) $115.7-\mathrm{keV}$, and (e) $174.8-\mathrm{keV} \gamma$-ray transitions. Vertical lines indicate $\alpha$-decay energies of interest (see text for details).

In addition to the aforementioned groups, the 5521- and $5571-\mathrm{keV}$ peaks are observed in coincidence with $E_{\gamma}=$ 472.1(5) and 421.4(5) $\mathrm{keV}$ transitions, respectively [see Figs. 6(f) and 6(g)]. ${ }^{2}$ The 5521-472.1 keV and 5571-421.4 $\mathrm{keV}$ groups both have $Q_{\alpha, t o t}=6120(10) \mathrm{keV}$, in good agreement with that of the 5925-56.8 keV decay path, establishing excited states at 472.1(3) and 421.4(3) keV above ${ }^{174} \mathrm{Ir}^{m}$, respectively (see Fig. 5).

The 5521- and 5571-keV $\alpha$ decays are also observed in coincidence with iridium $K_{\alpha, \beta}$ x rays. This is most likely due to yet unobserved and possibly strongly converted decays or cascades that feed to ${ }^{174} \mathrm{Ir}^{m}$ from the 472.1 and $421.4 \mathrm{keV}$ levels. There is some evidence that these two $\alpha$ lines are in coincidence with the $56.8-\mathrm{keV} \gamma$ transition, but the statistics are too low for certainty.

\footnotetext{
${ }^{2}$ The presence of these transitions was confirmed in a complementary high-statistics study performed using the JuroGam II-RITU-GREAT setup at JYFL [42].
}

\section{Multipolarity of transitions in ${ }^{174} \mathrm{Ir}^{m}$ and $\alpha-e^{-}$summing}

As in the case of ${ }^{178} \mathrm{Au}^{g}$, all the $\gamma$ transitions following the f.s. decay of ${ }^{178} \mathrm{Au}^{m}$ are prompt. This observation limits their multipolarities to $\Delta L \leqslant 2$.

The multipolarity of the $56.8-\mathrm{keV}$ transition was deduced by evaluating its total conversion coefficient, $\alpha_{\text {tot, exp }}$. This was calculated by comparing the number of singles $\alpha$ decays measured in the $5925 \mathrm{keV}$ peak $\left(N_{\alpha}\right)$ to the number of coincidence events in the 5925-56.8 keV $\alpha-\gamma$ group $\left(N_{\alpha, \gamma}\right)$, corrected for the detection efficiency of the 56.8-keV $\gamma$ ray $\left(\epsilon_{\gamma}\right)$. The total conversion coefficient is then given by

$$
\alpha_{\text {tot }, \exp }=\frac{N_{\alpha} \epsilon_{\gamma}}{N_{\alpha \gamma}}-1 .
$$

Using Eq. (2), a value of $\alpha_{\text {tot, exp }}(56.8 \mathrm{keV})=6.3(5)$ was deduced, which is closest to the theoretical value of $\alpha_{\text {tot }, t h}(56.8 ; M 1)=5.60(12)$ taken from BRICC. This agreement establishes an $M 1$ multipolarity for the $56.8-\mathrm{keV}$ transition, indicating that ${ }^{174} \mathrm{Ir}^{m}$ and the $56.8-\mathrm{keV}$ level have the same parity.

The high intensity of the $5925-\mathrm{keV} \alpha$ decay and the large value of $\alpha_{\text {tot,exp }}(56.8 \mathrm{keV})$ means a significant amount of $\alpha-e^{-}$ summing is present, whereby an $\alpha$ particle and a subsequent conversion electron are registered in the same silicon detector. This summing is of particular importance as the $L$-conversion electron for a 56.8-keV transition has an energy of $\approx 43 \mathrm{keV}$ [41], which, when summed to the $5925-\mathrm{keV} \alpha$ decay produces an $\approx 5968 \mathrm{keV}$ sum peak. Therefore, a significant fraction of the $5977-\mathrm{keV}$ peak in Fig. 3(b) could be due to $\alpha-e^{-}$ summing.

To understand the magnitude of this summing, GEANT4 $[43,44]$ simulations were performed. These included a 56.8$\mathrm{keV}$ transition with the corresponding conversion electron energies and intensities taken from BRICC, and iridium $L \mathrm{x}$ rays in the case of $L$ conversion. The blue histogram in Fig. 3(b) shows the results of the simulations and confirms that most of the intensity in the $5977-\mathrm{keV}$ peak is due to $\alpha-e^{-}$summing. The simulated spectrum was subtracted from the experimental data in order to evaluate the intensity $\left[I_{\alpha}=5.90(12) \%\right]$ and energy of the 5977-keV line.

The 5839-keV decay is seen in strong coincidence with iridium $K$ x rays. This can only be explained if the 91.2$\mathrm{keV}$ transition also has an $M 1$ multipolarity $\left(\alpha_{K, t h}(91.2)=\right.$ $6.57(11)$ [41]). This assignment is consistent with the number 5839-keV events in Fig. 3(b), and in the 5839-91.2 and 5839-56.8 keV coincidence groups, assuming that the 56.8- and 91.2-keV transitions are in cascade with each other.

No firm assignments for the multipolarities of the 421.4-, 472.1 -, and $139.2-\mathrm{keV}$ transitions can be made with the current statistics. However, the $\Delta L \leqslant 2$ limit allows us to place an upper limit of $<8 \%$ on the intensity of the $139.2-\mathrm{keV}$ transition, relative to the $91.2-56.8 \mathrm{keV}$ cascade.

\section{Half-lives, $\alpha$ - and $\beta$-branching ratios for ${ }^{178} \mathrm{Au}^{g, m}$}

The $\alpha$ - and $\beta$-decay branching ratio of ${ }^{178} \mathrm{Au}^{g}$ was calculated by comparing the number of $\alpha$ decays belonging 


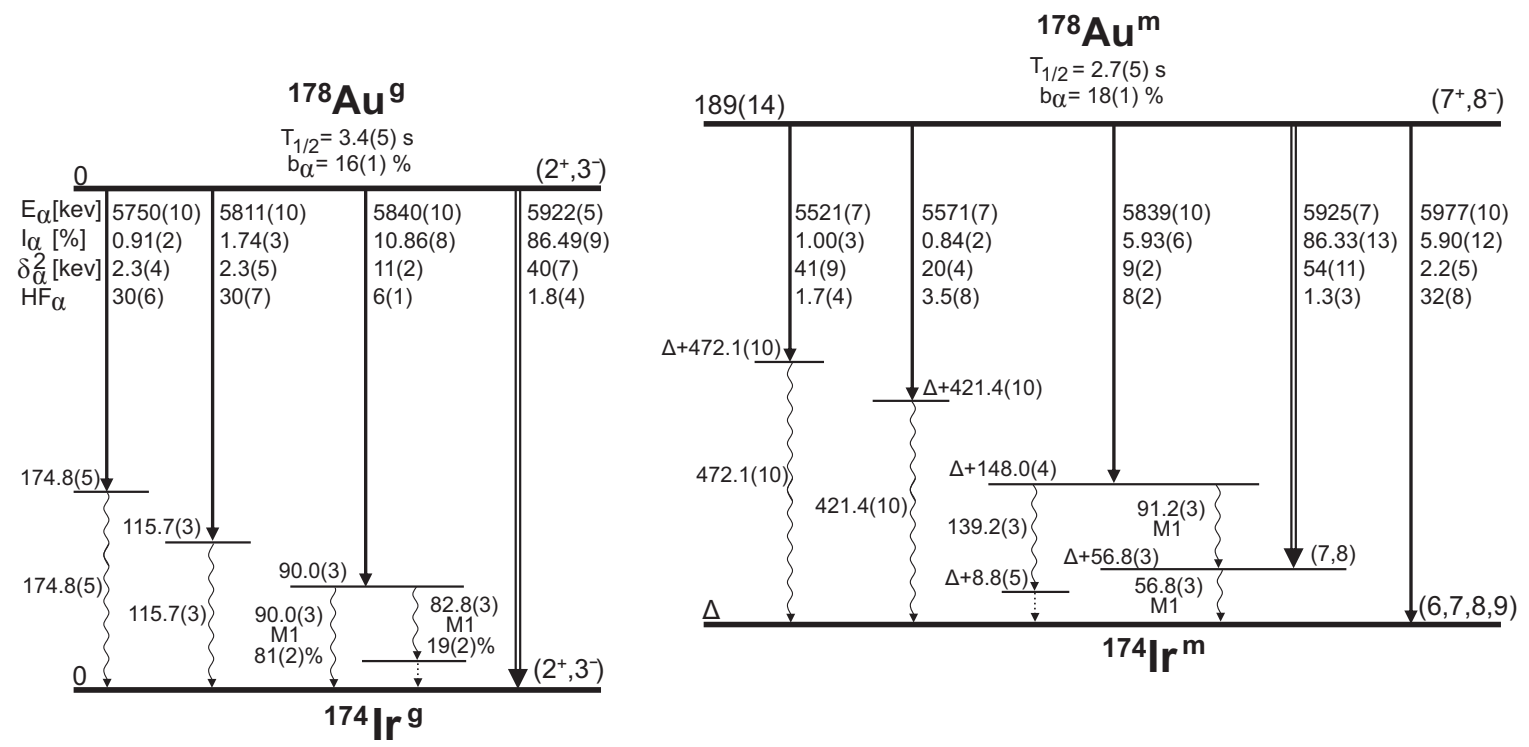

FIG. 5. Decay schemes of ${ }^{178} \mathrm{Au}^{g, m}$ deduced in this work. The $\alpha$-decay energies $\left(E_{\alpha}\right)$, intensities $\left(I_{\alpha}\right)$, reduced $\alpha$-decay widths $\left(\delta_{\alpha}^{2}\right)$, and hindrance factor values $\left(\mathrm{HF}_{\alpha}\right)$ are shown. The $\mathrm{HF}_{\alpha}$ values were calculated relative to the average $\delta_{\alpha}^{2}$ value for the unhindered $\alpha$ decays of ${ }^{177} \mathrm{Au}$ $\left(11 / 2^{-} \rightarrow 11 / 2^{-}, \delta_{\alpha}^{2}=82(14) \mathrm{keV}[39]\right)$ and ${ }^{179} \mathrm{Au}\left(1 / 2^{+} \rightarrow 1 / 2^{+}, \delta_{\alpha}^{2}=58(4) \mathrm{keV}\right.$ [40]). A value of $\Delta=129(17) \mathrm{keV}$ is determined, based on the decay data and mass measurements presented in the current work.

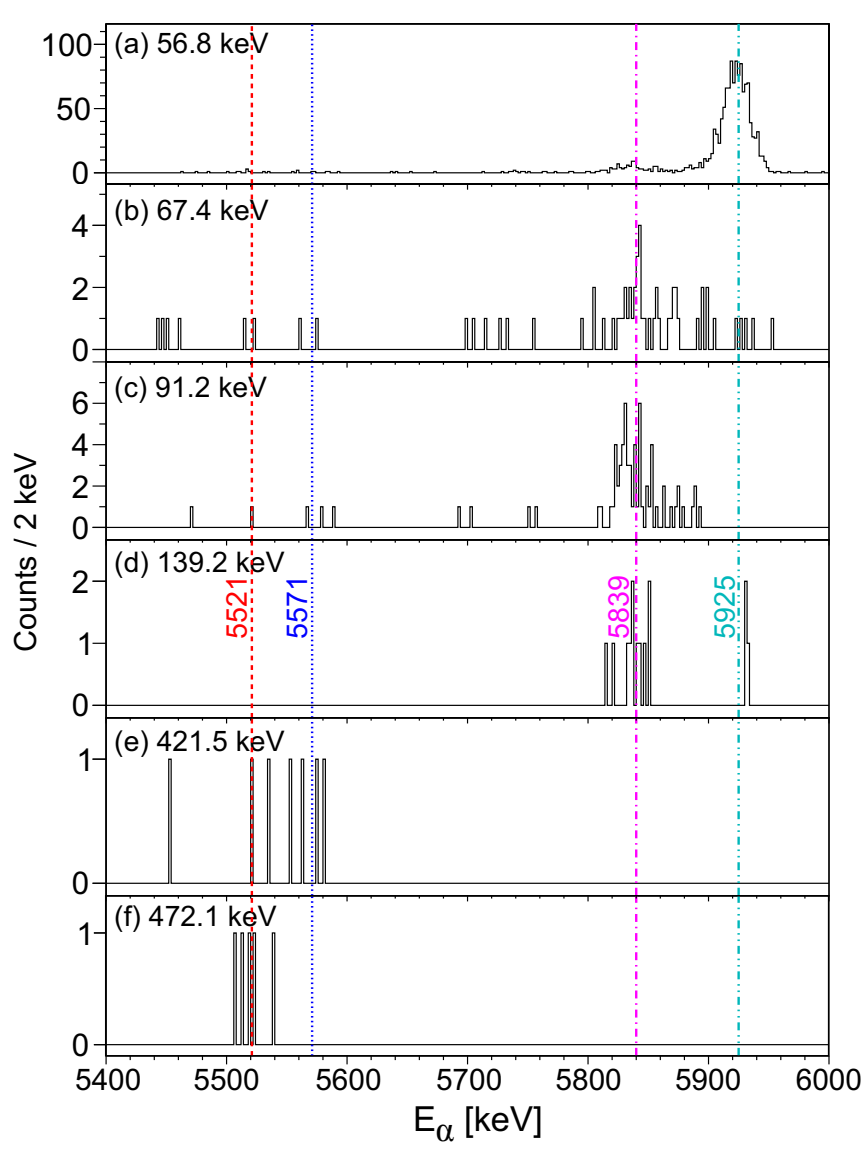

FIG. 6. Projections on the $E_{\alpha}$ axis for the $\alpha-\gamma$ groups of ${ }^{178} \mathrm{Au}^{m}$ shown in Fig. 3(c), for the (a) 56.8-keV, (b) 67.4-keV, (c) $91.2-\mathrm{keV}$, (d) $139.2-\mathrm{keV}$, (e) $421.5-\mathrm{keV}$, and (f) 472.1-keV $\gamma$-ray transitions. Vertical lines indicate $\alpha$-decay energies of interest (see text for details). to ${ }^{178} \mathrm{Au}$ and ${ }^{178} \mathrm{Pt}$ in Fig. 3(a), corrected for $b_{\alpha}\left({ }^{178} \mathrm{Pt}\right)=$ $7.7(3) \%[45]$, such that

$$
\begin{aligned}
b_{\alpha}\left({ }^{178} \mathrm{Au}^{g}\right) & =\frac{N_{\alpha}\left({ }^{178} \mathrm{Au}^{g}\right)}{N_{\alpha}\left({ }^{178} \mathrm{Au}^{g}\right)+N_{\beta}\left({ }^{178} \mathrm{Au}^{g}\right)} \\
& =\frac{N_{\alpha}\left({ }^{178} \mathrm{Au}^{g}\right)}{N_{\alpha}\left({ }^{178} \mathrm{Au}^{g}\right)+\frac{N_{\alpha}\left({ }^{178} \mathrm{Pt}\right)}{b_{\alpha}\left({ }^{178} \mathrm{Pt}\right)}} .
\end{aligned}
$$

The same approach was used for calculating $b_{\alpha}\left({ }^{178} \mathrm{Au}^{m}\right)$. This method assumes negligible ionization of ${ }^{178} \mathrm{Pt}$ in the ion source due to it being a refractory element, which was confirmed by measurements made with the lasers turned off.

Data were taken from runs in which the WM wheel was not rotated, to avoid removing any ${ }^{178} \mathrm{Au}$ or ${ }^{178} \mathrm{Pt}$ activity. The extracted values were $b_{\alpha}=16(1) \%, b_{\beta}=84(1) \%$ for ${ }^{178} \mathrm{Au}^{g}$, and $b_{\alpha}=18(1) \%, b_{\beta}=82(1) \%$ for ${ }^{178} \mathrm{Au}^{m}$. We note that these values are significantly different to the $b_{\alpha} \geqslant 40 \%$ of Ref. [23]. This discrepancy is most likely due to contamination from unwanted reaction channels in [23].

Half-life measurements for ${ }^{178} \mathrm{Au}^{g, m}$ were performed using a grow-in/decay method which involved cycles of $13.2 \mathrm{~s}$ of pulsed implantation, followed by a $6 \mathrm{~s}$ decay period. The data were corrected for the dead time of the system, which was monitored by using the recorded count rate of a pulser. Figure 7 shows the decay curves extracted by counting the number of $\alpha$ decays, along with exponential fits of the data. From these fits, values of $T_{1 / 2}\left({ }^{178} \mathrm{Au}^{g}\right)=3.4(5) \mathrm{s}$ and $T_{1 / 2}\left({ }^{178} \mathrm{Au}^{m}\right)=2.7(5) \mathrm{s}$ were extracted. Conservative error estimates are given due to the dead time correction and a short measurement time relative to the extracted values. 


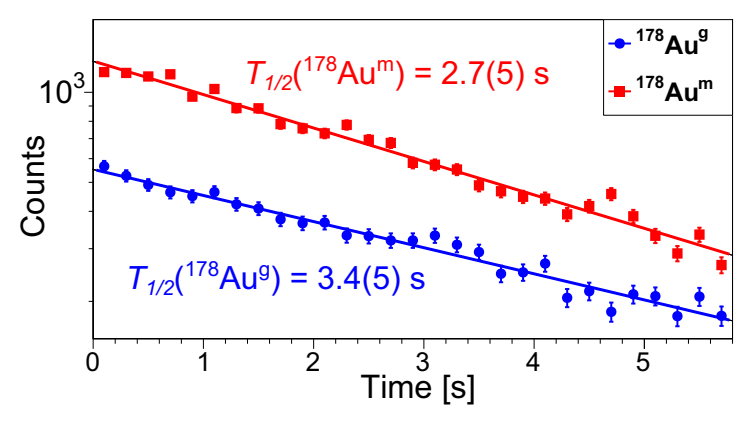

FIG. 7. The decay curves for ${ }^{178} \mathrm{Au}^{g}(\bullet)$ and ${ }^{178} \mathrm{Au}^{m}(\square)$, fitted with exponential functions.

\section{Fine structure $\alpha$ decay of ${ }^{178} \mathrm{Pt}$}

In Figs. 3(a) and 3(b), the $\alpha$ decays of ${ }^{178} \mathrm{Pt}$ are clearly seen at 5446 and $5291 \mathrm{keV}$. The intensities of the two peaks were evaluated by fitting the singles data with Crystal Ball functions. From the results of the fit, values of $I_{\alpha}(5291)=$ $2.6(1) \%$ and $I_{\alpha}(5446)=97.4(1) \%$ were deduced. These values agree with the currently accepted values of $I_{\alpha}(5291)=$ $5.1(24) \%$ and $I_{\alpha}(5446)=94.9(24) \%$ [46], but are more precise.

\section{Spin assignments for ${ }^{178} \mathrm{Au}^{g, m}$}

The possible spin assignments for ${ }^{178} \mathrm{Au}^{g, m}$ can be explored by using two complementary methods. In the first approach we consider the $\beta$-decay feeding pattern to states in the daughter nucleus, ${ }^{178} \mathrm{Pt}$, and in the second we compare the relative intensities of the hfs components shown in Fig. 1(b) with theoretical transition strengths.

\section{1. $\beta$-decay feeding pattern}

Figures 8 (a) and 8 (b) show the background-subtracted singles $\gamma$-ray spectra, following the $\beta^{+} / \mathrm{EC}$ decay of ${ }^{178} \mathrm{Au}^{g}$ and ${ }^{178} \mathrm{Au}^{m}$, respectively. The background spectra were taken during runs with the lasers switched off.

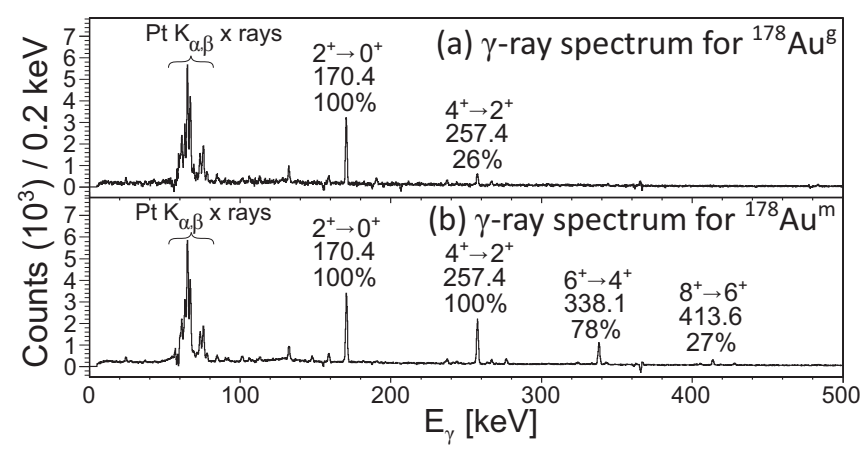

FIG. 8. Background-subtracted singles $\gamma$-decay spectra measured by the LEGe following the $\beta^{+} /$EC decays of (a) ${ }^{178} \mathrm{Au}^{g}$ and (b) ${ }^{178} \mathrm{Au}^{m}$. The relevant peaks are labeled with their energies (in $\mathrm{keV}$ ), along with the spins and parities of the initial and final states in ${ }^{178} \mathrm{Pt}$ and their intensities corrected for $\gamma$-ray detection efficiency, normalized to the intensity of the $170.4-\mathrm{keV}^{+} \rightarrow 0^{+}$transition.
The 257.4(3)- and 170.4(3)-keV $\gamma$ lines in Fig. 8(a), recorded with RILIS settings for ${ }^{178} \mathrm{Au}^{g}$, correspond to the known $4_{1}^{+} \rightarrow 2_{1}^{+}$and $2_{1}^{+} \rightarrow 0_{1}^{+} E 2$ transitions within the ground-state band of ${ }^{178} \mathrm{Pt}$. From these observations, and assuming no high-order forbidden decays between ${ }^{178} \mathrm{Au}$ and ${ }^{178} \mathrm{Pt}$, the possible assignments for the spin of ${ }^{178} \mathrm{Au}^{g}$ may be restricted to $I=(2,3)$. An $I=4$ assignment is unlikely as a preferential feeding to the yrast $2^{+}$state in ${ }^{178} \mathrm{Pt}$ is observed. Indeed, the low intensity of the 257.4-keV peak in Fig. 8(a) could indicate that there is no direct feeding to the $4^{+}$state in ${ }^{178} \mathrm{Pt}$, in which case all counts in this peak would be due to the Pandemonium effect [47].

For the ${ }^{178} \mathrm{Au}^{m}$ case, four $\gamma$ lines at $E_{\gamma}=413.6(3)$, 338.1(3), 257.4(3) and 170.4(3) keV are seen in Fig. 8(b), establishing feeding up to the $8^{+}$member of the ground-state band in ${ }^{178} \mathrm{Pt}$. Based on this, the spin assignment for ${ }^{178} \mathrm{Au}^{m}$ may be restricted to 7 or 8 , as no feeding to $I=10$ states is observed.

Despite their inability to separate the two isomers, Davidson et al. made similar observations (see Fig. 7 in Ref. [24]), which showed a feeding of states in ${ }^{178} \mathrm{Pt}$ was consistent with the presence of a low- and a high-spin isomer in ${ }^{178} \mathrm{Au}$, with $I \approx 2$ and $\approx 8$, respectively.

\section{Relative intensities of hfs components}

Due to the low angular momenta of the electronic states involved in the scanned transition, it is impossible to determine the nuclear spin by simply counting the number of hfs components [48]. Nevertheless, information about the nuclear spin can be obtained by comparing the experimental intensity ratios of the hfs components to the theoretical ones that are dependent on the nuclear spin (see corresponding formulas, for example, in Ref. [49]). However, saturation of the first excitation step markedly distorts the shape of the hfs spectrum. Therefore, this method cannot be applied in our case since some degree of saturation was present during our experiment in order to increase the count rate for isotopes with low yields. Instead, one should consider the ratio of the sums of the amplitudes of the transitions from each hfs sublevel of the atomic ground state:

$$
R(I)=\frac{\Gamma_{F_{1} \rightarrow F_{1}^{\prime}}+\Gamma_{F_{1} \rightarrow F_{2}^{\prime}}}{\Gamma_{F_{2} \rightarrow F_{1}^{\prime}}+\Gamma_{F_{2} \rightarrow F_{2}^{\prime}}}=\frac{2 F_{1}+1}{2 F_{2}+1}=\frac{I+1}{I},
$$

where $\Gamma$ is the theoretical transition strength between states $F$ and $F^{\prime}$. Hence, the theoretically expected ratios are $R(1)=2$, $R(2)=1.5, R(3)=1.33$, and $R(4)=1.25$.

In the case of ${ }^{178} \mathrm{Au}^{g}$, the experimental ratio $\left(R_{\text {exp }}\right)$ is equal to the integrated intensity ratio of peaks 3 and 4 in Fig. 1(b), which was assessed using a Simpson integration method in order to remove any effects due to non-uniform intervals between laser frequency steps. This ratio only weakly depends on laser saturation and other distorting effects on the spectrum [50]. A weighted mean of the three hfs scans yielded a value of $R_{\exp }=1.51(13)$, in good agreement with the theoretical value $R(2)=1.5$, suggesting a most probable assignment of $I\left({ }^{178} \mathrm{Au}^{g}\right)=2$. However, $I\left({ }^{178} \mathrm{Au}^{g}\right)=3$ cannot be ruled out with certainty, as the $R_{\text {exp }}$ value differs from $R(3)$ by less than 
TABLE II. Comparison of experimental results for the magnetic dipole moments, $\mu_{\mathrm{exp}}$, of ${ }^{178} \mathrm{Au}^{g, m}$ for different nuclear spin assumptions, with those calculated for specific Nilsson configurations using the deformed additivity rule, $\mu_{\text {add }}$.

\begin{tabular}{llrrrrrrr}
\hline \hline State & $I^{\pi}$ & $a(6 s)(\mathrm{MHz})$ & $a(6 p) / a(6 s)$ & ${ }^{197} \Delta^{178}(\%)$ & $\mu_{\exp }\left(\mu_{N}\right)^{\mathrm{a}}$ & $\mu_{\exp }\left(\mu_{N}\right)^{\mathrm{b}}$ & $\mu_{\text {add }}\left(\mu_{N}\right)$ & Nilsson configuration \\
\hline${ }^{178} \mathrm{Au}^{g}$ & $2^{+}$ & $-12760(300)$ & $0.1122(60)$ & $8.7(79)$ & $-0.880(56)$ & $-0.884(68)$ & $-0.73(20)$ & $\pi 1 / 2^{-}[541]_{h_{9 / 2}} \otimes \nu 5 / 2^{-}[512]_{h_{9 / 2}}$ \\
& $3^{-}$ & $-9100(300)$ & $0.1136(60)$ & $10.6(80)$ & $-0.941(69)$ & $-0.962(77)$ & $-0.74(21)$ & $\pi 1 / 2^{-}[541]_{h_{9 / 2}} \otimes \nu 7 / 2^{+}[633]_{i_{13 / 2}}$ \\
${ }^{178} \mathrm{Au}^{m}$ & $7^{+}$ & $19602(40)$ & $0.1137(13)$ & $10.7(19)$ & $4.74(20)$ & $4.84(8)$ & $4.67(34)$ & $\pi 9 / 2^{-}[514]_{h_{11 / 2}} \otimes 5 / 2^{-}[512]_{h_{9 / 2}}$ \\
& $8^{-}$ & $17297(40)$ & $0.1137(13)$ & $10.6(18)$ & $4.78(20)$ & $4.89(8)$ & $4.84(33)$ & $\pi 9 / 2^{-}[514]_{h_{11 / 2}} \otimes \nu / 2^{+}[633]_{i_{13 / 2}}$ \\
& $8^{+}$ & $17297(40)$ & $0.1137(13)$ & $10.6(18)$ & $4.78(20)$ & $4.89(8)$ & $5.61(34)$ & $\pi 11 / 2^{-}[505]_{h_{11 / 2}} \otimes \nu 5 / 2^{-}[512]_{h_{9 / 2}}$ \\
\hline
\end{tabular}

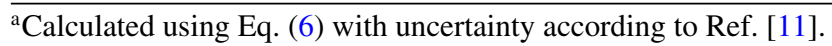

${ }^{\mathrm{b} C a l c u l a t e d}$ using Eq. (7), accounting for the hfa using the procedure proposed in Ref. [20].

$1.3 \sigma$. In either case, the assignments are consistent with the observed $\beta$-decay feeding pattern.

\section{E. Magnetic dipole moments for ${ }^{178} \mathrm{Au}^{g, m}$}

The positions of the hfs components as a function of laser frequency are defined as

$$
v_{F, F^{\prime}}=v_{0}+a(6 p) \frac{K^{\prime}}{2}-a(6 s) \frac{K}{2},
$$

where $v_{0}$ is the center of gravity of the hfs, $K=F(F+1)-$ $I(I+1)-J(J+1)$, the prime symbols denote the upper level of the atomic transition and $a(n \ell)$ is the hyperfine coupling constant for the atomic level with quantum numbers $n$ and $\ell$. The hfs spectra were fitted with Voigt profiles, with assumptions of $I\left({ }^{178} \mathrm{Au}^{g}\right)=2$ or 3 and $I\left({ }^{178} \mathrm{Au}^{m}\right)=7$ or 8 . The $a(6 s)$ values extracted from the fitting procedure are given in Table II, for the different spin assumptions.

The magnetic moments were determined using two approaches. The first used the same approach as Ekström et al. [11], as modified in [51], such that

$$
\mu_{\mathrm{exp}}=\frac{a(6 s) I}{29005} \mu_{N} .
$$

This relation accounts for the hyperfine anomaly (hfa) by application of the empirical Moskowitz-Lombardi rule [52].

However, it was recently shown that the assumption concerning the hfa made by Ekström et al. is not well justified [20]. Alternatively, the $\mu_{\text {exp }}$ can be calculated more accurately using the expression

$$
\mu_{\mathrm{exp}}=\mu_{\mathrm{ref}} \frac{I_{A}}{I_{\text {ref }}} \frac{a_{A}}{a_{\text {ref }}}\left(1+{ }^{\mathrm{ref}} \Delta^{A}\right),
$$

where "ref" denotes a reference isotope $\left({ }^{197} \mathrm{Au}\right)$ with known $\mu$ and $a$ values, and ${ }^{\text {ref }} \Delta^{A}$ is the relative hfa.

It was shown in Ref. [20] that the relative hfa could be determined by comparing the ratio of the hyperfine constants of different atomic states, for different nuclei. In the present work, this was done using the ratio $a(6 p) / a(6 s)$ for gold isotopes (the same approach was used in Refs. [53-55] for gallium, thallium, and bismuth isotopes; see there for details). The values for the $a(6 p) / a(6 s)$ ratios along with the $\mu_{\exp }$ values determined using both approaches are given in Table II.

\section{DISCUSSION}

\section{A. Configuration assignments for ${ }^{178} \mathrm{Au}^{g, m}$}

The ground and isomeric states of ${ }^{183-186} \mathrm{Au}(N=$ 104-107) are well deformed and presumably prolate $[11,12,51,56-60]$. Thus, the ground states of these isotopes are well described by configurations based on deformed $\pi 3 / 2^{-}[532]$ and/or $\pi 1 / 2^{-}$[541] Nilsson proton states as opposed to spherical $\pi 3 s_{1 / 2} / \pi 2 d_{3 / 2}$ orbitals in heavier gold nuclei with $N>107$. The nucleus ${ }^{178} \mathrm{Au}(N=99)$ lies just beyond the expected end of this region of strong deformation [21]. However, isotope shift measurements taken during the same experiment as the present work show that both ${ }^{178} \mathrm{Au}^{g, m}$ are deformed, with $\left\langle\beta_{2}{ }^{2}\right\rangle^{1 / 2} \approx 0.25$ and $\approx 0.3$, respectively [61]. We will consider both states to be prolate, as the ${ }^{177} \mathrm{Pt}$ core is known to have a large positive deformation [62].

Based on these $\beta_{2}$ values, we will only consider the systematics of the low-lying deformed states near $Z=79$ and $N=$ 99 when exploring the possible configurations of ${ }^{178} \mathrm{Au}^{g, m}$. As seen in Fig. 8 of Ref. [18], there are many proton and neutron states lying close to the Fermi surface of ${ }^{178} \mathrm{Au}$ at $\beta_{2} \approx 0.25$. All of these orbitals were considered for the odd particle states in our analysis (see Ref. [18] for details).

The possible configurations may be constrained by using the Gallagher-Moszkowski rules [63] to predict which Nilsson orbitals near the Fermi surface are most likely to couple to $I\left({ }^{178} \mathrm{Au}^{g}\right)=2,3$ and $I\left({ }^{178} \mathrm{Au}^{m}\right)=7,8$. The deformed additivity rule [64] can then be used to calculate the expected magnetic dipole moments, $\mu_{\text {add }}$, for these configurations. As single-particle $g$ factors are needed for the application of the additivity relation, experimental values for the chosen orbitals were used. When such data are unavailable, $g$ factors were calculated by the standard Nilsson-model approach with different parameter sets [65-67]. This is the same approach as used in Ref. [18] for ${ }^{180,182} \mathrm{Au}$; see therein for more details. The calculated $\mu_{\text {add }}$ values are compared with the experimental results from the present work, $\mu_{\exp }$, in Table II.

For ${ }^{178} \mathrm{Au}^{m}$, a good agreement between $\mu_{\text {exp }}$ and $\mu_{\text {add }}$ is seen for both the $\pi 9 / 2^{-}[514] \otimes \nu 5 / 2^{-}[512]\left(I^{\pi}=\right.$ $\left.7^{+}\right)$and $\pi 9 / 2^{-}[514] \otimes \nu 7 / 2^{+}[633]\left(I^{\pi}=8^{-}\right)$configurations, whereas a reasonable agreement for $\pi 1 / 2^{-}[541] \otimes$ $v 5 / 2^{-}[512]\left(I^{\pi}=2^{+}\right)$and $\pi 1 / 2^{-}[541] \otimes v 7 / 2^{+}[633]\left(I^{\pi}=\right.$ $3^{-}$) is seen for ${ }^{178} \mathrm{Au}^{g}$. The systematics of the ground states for $N=99$ nuclei shows that both $v 7 / 2^{+}[633]$ and $v 5 / 2^{-}[512]$ 
states may be occupied by the 99th neutron, with $v 7 / 2^{+}[633]$ being the case for the $Z=66,68,70,72$ isotones, and the $v 5 / 2^{-}$[512] for $Z=74,76,78$ [46]. The inversion of these orbitals coincides with a change in deformation; ${ }_{66}^{165} \mathrm{Dy},{ }_{68}^{167} \mathrm{Er}$, ${ }_{70}^{169} \mathrm{Yb},{ }_{72}^{171} \mathrm{Hf}$ have $\beta_{2} \approx 0.3$, whereas ${ }_{74}^{173} \mathrm{~W},{ }_{76}^{175} \mathrm{Os},{ }_{78}^{177} \mathrm{Pt}$ have $\beta_{2} \approx 0.25$. Correspondingly, in ${ }^{178} \mathrm{Au}^{g}$ with $\beta_{2} \approx 0.25$ one may expect that the odd neutron occupies the $v 5 / 2^{-}[512]$ state, and hence $I^{\pi}\left({ }^{178} \mathrm{Au}^{g}\right)=2^{+}$seems more likely. Similarly, in ${ }^{178} \mathrm{Au}^{m}$ with $\beta_{2} \approx 0.3$, a $v 7 / 2^{+}[633]$ odd neutron and $I^{\pi}\left({ }^{178} \mathrm{Au}^{m}\right)=8^{-}$is preferable. However, we note that both a $\pi 9 / 2^{-}[514] \otimes \nu 5 / 2^{-}[512]$ and $\pi 9 / 2^{-}[514] \otimes \nu 7 / 2^{+}[633]$ band were observed in isotonic ${ }^{176} \mathrm{Ir}$, with low-lying $I^{\pi}=7^{+}$ and $I^{\pi}=8^{-}$bandheads [46].

\section{B. Reduced $\alpha$-decay widths}

Reduced $\alpha$-decay widths $\left(\delta_{\alpha}^{2}\right)$ were calculated using the Rasmussen approach [68], assuming $\Delta L=0$ decays. The results of these calculations are shown in Fig. 5, along with hindrance factors $\left(\mathrm{HF}_{\alpha}\right)$ calculated relative to the average $\delta_{\alpha}^{2}$ value of the unhindered $\alpha$ decays in neighboring ${ }^{177} \mathrm{Au}\left(11 / 2^{-} \rightarrow 11 / 2^{-}, \delta_{\alpha}^{2}=82(14) \mathrm{keV}\right.$ [39] $)$ and ${ }^{179} \mathrm{Au}$ $\left(1 / 2^{+} \rightarrow 1 / 2^{+}, \delta_{\alpha}^{2}=58(4) \mathrm{keV}\right.$ [40]).

The unhindered 5922-keV decay with $\delta_{\alpha}^{2}=40(7) \mathrm{keV}$ suggests that ${ }^{178} \mathrm{Au}^{g}$ and ${ }^{174} \mathrm{Ir}^{g}$ have the same nuclear spin and configuration. This would mean a preferred $I^{\pi}=\left(2^{+}, 3^{-}\right)$ assignment for ${ }^{174} \operatorname{Ir}^{g}$ rather than the tentative $I^{\pi}=\left(3^{+}\right)$proposed in Ref. [25], which was based on the observed $\beta$-decay feeding pattern to states in ${ }^{174}$ Os.

Using similar arguments, based on the $I=(7,8)$ assignment for ${ }^{178} \mathrm{Au}^{m}$ and the unhindered nature of the 5925-keV $\alpha$ decay, the $56.8 \mathrm{keV}$ state above ${ }^{174} \mathrm{Ir}^{m}$ should also have $I=(7,8)$. Thus, the $M 1$ multipolarity of the 56.8-keV transition constrains the possible spin of ${ }^{174} \mathrm{Ir}^{m}$ to $I=6-9$. This agrees with $I^{\pi}\left({ }^{174} \operatorname{Ir}^{m}\right)=\left(7^{+}\right)$proposed in Ref. [25].

Along with the 5925-keV decay, the 5521- and 5571-keV $\alpha$ lines of ${ }^{178} \mathrm{Au}^{m}$ are also unhindered $\left[\mathrm{HF}_{\alpha}=1.7(4)\right.$ and 3.5(8), respectively]. The low hindrance could indicate that the configurations of the 472.1-, 421.4-, and 56.8-keV states are similar, and/or possibly mixed. Alternatively, this observation could be due to these three $\alpha$ decays feeding to members of the same rotational band in the daughter nucleus. However, not enough is known about the structure of ${ }^{174}$ Ir to confirm or contradict either suggestion.

We note that it is unusual to have several unhindered $\alpha$ decays from one nucleus. Indeed, odd-odd nuclei above $Z=$ 82 typically have several strongly hindered decays and just one unhindered decay [69].

\section{Determination of the $\Delta$ value and possible evidence of an isomer in ${ }^{170} \mathrm{Re}$}

The value of $\Delta=129(17) \mathrm{keV}$ determined in the present work for the excitation energy of ${ }^{174} \mathrm{Ir}^{m}$ is significantly different from the previous value of 193(11) keV in Ref. [25]. However, apparently this latter excitation energy was deduced based on the assumption that the f.s. decays of both ${ }^{174} \operatorname{Ir}^{g, m}$ eventually feed to the ground state in ${ }^{170} \operatorname{Re}$ (see Fig. 7 of [25]). The justification for this assumption was not explained in the original publication.

Assuming that the $\alpha-\gamma$ coincidences decay of Ref. [25] are correct, the 64(20) keV difference between our and the previous excitation energy of ${ }^{174} \mathrm{Ir}^{m}$ indicates that the assumption of feeding to ${ }^{170} \mathrm{Re}^{g}$ is likely incorrect. In which case, the f.s. decays of ${ }^{174} \mathrm{Ir}^{g}$ actually feed to an excited state at $64(20) \mathrm{keV}$ in ${ }^{170} \mathrm{Re}$, providing evidence for a previously unknown isomer. In this scenario, the decay of ${ }^{174} \mathrm{Ir}^{g}$ feeds to ${ }^{170} \mathrm{Re}^{m}$, and ${ }^{174} \mathrm{Ir}^{m}$ to ${ }^{170} \operatorname{Re}^{g}$.

\section{CONCLUSION}

By using selective experimental techniques, it was possible to identify and produce isomerically pure beams of two $\alpha$ decaying states in the neutron-deficient nucleus, ${ }^{178} \mathrm{Au}$. Using the RILIS, the Windmill decay station, the ISOLTRAP Penning trap, and MR-ToF MS, independent measurements of the masses, nuclear decay properties and hyperfine structures of the two states were performed. Detailed decay schemes for the two states were deduced, and preferred spin, parity, and configuration assignments of $I^{\pi}=2^{+}, \pi 1 / 2^{-}[541] \otimes$ $\nu 5 / 2^{-}[512]$ or $I^{\pi}=3^{-}, \pi 1 / 2^{-}[541] \otimes \nu 7 / 2^{+}[633]$ for ${ }^{178} \mathrm{Au}^{g}$, and either $I^{\pi}=7^{+}, \pi 9 / 2^{-}[514] \otimes \nu 5 / 2^{-}[512]$ or $I^{\pi}=8^{-}, \pi 9 / 2^{-}[514] \otimes \nu 7 / 2^{+}[633]$ for ${ }^{178} \mathrm{Au}^{m}$ are proposed. The $\delta_{\alpha}^{2}$ values for ${ }^{178} \mathrm{Au}^{m}$ provide surprising evidence for several unhindered $\alpha$ decays belonging to this nuclide. The results from the decay data and the mass measurements of the present work provide the first information on low-lying excited states in ${ }^{174} \mathrm{Ir}$, and may indicate the presence of a previously unknown isomeric state in ${ }^{170} \mathrm{Re}$.

\section{ACKNOWLEDGMENTS}

We would like to acknowledge the support of the ISOLDE Collaboration and technical teams. This work was done with support from European Union's Seventh Framework Programme for Research and Technological Development under Grants Agreement No. 262010 (ENSAR), No. 267194 (COFUND), and No. 289191 (LA3NET); from the European Union's Horizon 2020 Framework Research and Innovation Programme under Grant Agreement No. 654002 (ENSAR2); from the U.K. Science and Technology Facilities Council Grant ST/P003885/1; from the BMBF (05P12HGCI1, 05P12HGFNE, 05P15ODCIA, and 05P15HGCIA); from the Max Planck Society; from the French IN2P3; from FWOVlaanderen (Belgium) by GOA/2010/010 (BOF KU Leuven); from the Interuniversity Attraction Poles Programme initiated by the Belgian Science Policy Office (BriX network P7/12); from RFBR according to the research Project No. 19-02-00005; from the Slovak Research and Development Agency (Contract No. APVV-14-0524) and the Slovak Grant Agency VEGA (Contract No. 1/0532/17). A.W. acknowledges support by a Wolfgang Gentner Ph.D. Scholarship of the BMBF (05E15CHA). 
[1] B. A. Marsh et al., Nucl. Instrum. Methods Phys. Res., Sect. B 317, 550 (2013).

[2] Y. Kudryavtsev et al., Nucl. Instrum. Methods Phys. Res., Sect. B 114, 350 (1996).

[3] I. D. Moore et al., in Frontiers in Nuclear Structure, Astrophysics, and Reactions - FINUSTAR, 12-17 September 2005, edited by S. V. Harissopulos, P. Demetriou, and R. Julin, AIP Conf. Proc. No. 831 (AIP, New York, 2006), pp. 511-513.

[4] J. Lassen et al., in 4th International Conference on Laser Probing - LAP 2008, 6-10 October 2008, Nagoya, Japan, edited by T. Iguchi and K. Watanabe, AIP Conf. Proc. No. 1104 (AIP, New York. 2009), pp. 9-15.

[5] J. Van Roosbroeck et al., Phys. Rev. Lett. 92, 112501 (2004).

[6] V. N. Fedosseev, Y. Kudryavtsev, and V. I. Mishin, Phys. Scr. 85, 058104 (2012).

[7] I. Stefanescu et al., Phys. Rev. Lett. 98, 122701 (2007).

[8] M. Venhart, J. L. Wood, M. Sedlák, M. Balogh, M. Bírová, A. J. Boston, T. E. Cocolios, L. J. Harkness-Brennan, R.-D. Herzberg, L. Holub, D. T. Joss, D. S. Judson, J. Kliman, J. Klimo, L. Krupa, J. Lušnák, L. Makhathini, V. Matoušek, Š. Motyčák, R. D. Page, A. Patel, K. Petrík, A. V. Podshibyakin, P. M. Prajapati, A. M. Rodin, A. Špaček, R. Urban, C. Unsworth, and M. Veselský, J. Phys. G: Nucl. Part. Phys. 44, 074003 (2017).

[9] M. Sedlák et al., Eur. Phys. J. A 56, 161 (2020).

[10] M. Venhart, M. Balogh, A. Herzáñ, J. Wood, F. Ali, D. Joss, A. Andreyev, K. Auranen, R. Carroll, M. Drummond, J. Easton, P. Greenlees, T. Grahn, A. Gredley, J. Henderson, U. Jakobsson, R. Julin, S. Juutinen, J. Konki, E. Lawrie, M. Leino, V. Matoušek, C. McPeake, D. O’Donnell, R. Page, J. Pakarinen, P. Papadakis, J. Partanen, P. Peura, P. Rahkila, P. Ruotsalainen, M. Sandzelius, J. Sarén, B. Saygl, M. Sedlák, C. Scholey, J. Sorri, S. Stolze, A. Thornthwaite, R. Urban, J. Uusitalo, M. Veselský, and F. Wearing, Phys. Lett. B 806, 135488 (2020).

[11] C. Ekström, L. Robertsson, S. Ingelman, G. Wannberg, and I. Ragnarsson, Nucl. Phys. A 348, 25 (1980).

[12] K. Wallmeroth, G. Bollen, A. Dohn, P. Egelhof, J. Grüner, F. Lindenlauf, U. Krönert, J. Campos, A. Rodriguez Yunta, M. J. G. Borge, A. Venugopalan, J. L. Wood, R. B. Moore, and H. J. Kluge, Phys. Rev. Lett. 58, 1516 (1987).

[13] W. F. Mueller et al., Phys. Rev. C 59, 2009 (1999).

[14] F. G. Kondev et al., Phys. Lett. B 512, 268 (2001).

[15] W. F. Mueller et al., Phys. Rev. C 69, 064315 (2004).

[16] M. Venhart et al., Phys. Lett. B 695, 82 (2011).

[17] J. G. Cubiss et al., Phys. Lett. B 786, 355 (2018).

[18] R. D. Harding et al., Phys. Rev. C 102, 024312 (2020).

[19] A. E. Barzakh et al., Phys. Rev. C 101, 064321 (2020).

[20] A. E. Barzakh et al., Phys. Rev. C 101, 034308 (2020).

[21] J. L. Wood, E. F. Zganjar, C. De Coster, and K. Heyde, Nucl. Phys. A 651, 323 (1999).

[22] A. Siivola, Nucl. Phys. A 109, 231 (1968).

[23] J. G. Keller et al., Nucl. Phys. A 452, 173 (1986).

[24] P. M. Davidson et al., Nucl. Phys. A 657, 219 (1999).

[25] W.-D. Schmidt-Ott, H. Salewski, F. Meissner, U. Bosch-Wicke, P. Koschel, V. Kunze, and R. Michaelsen, Nucl. Phys. A 545, 646 (1992).

[26] V. Fedosseev, K. Chrysalidis, T. D. Goodacre, B. Marsh, S. Rothe, C. Seiffert, and K. Wendt, J. Phys. G: Nucl. Part. Phys. 44, 084006 (2017).

[27] R. Catherall, W. Andreazza, M. Breitenfeldt, A. Dorsival, G. J. Focker, T. P. Gharsa, T. J. Giles, J.-L. Grenard, F. Locci,
P. Martins, S. Marzari, J. Schipper, A. Shornikov, and T. Stora, J. Phys. G: Nucl. Part. Phys. 44, 094002 (2017).

[28] V. I. Mishin, V. N. Fedoseyev, H.-J. Kluge, V. S. Letokhov, H. L. Ravn, F. Scheerer, Y. Shirakabe, S. Sundell, and O. Tengblad, Nucl. Instrum. Methods Phys. Res., Sect. B 73, 550 (1993).

[29] M. Mukherjee, D. Beck, K. Blaum, G. Bollen, J. Dilling, S. George, F. Herfurth, A. Herlert, A. Kellerbauer, H. J. Kluge, S. Schwarz, L. Schweikhard, and C. Yazidjian, Eur. Phys. J. A 35, 1 (2008)

[30] R. N. Wolf et al., Int. J. Mass Spectrom. Ion Processes 349-350, 123 (2013).

[31] A. N. Andreyev et al., Phys. Rev. Lett. 105, 252502 (2010).

[32] M. D. Seliverstov et al., Phys. Rev. C 89, 034323 (2014).

[33] A. Rytz, At. Data Nucl. Data Tables 47, 205 (1991).

[34] J. G. Cubiss et al., Phys. Rev. C 97, 054327 (2018).

[35] M. König et al., Int. J. Mass Spectrom. Ion Processes 142, 95 (1995).

[36] M. Wang et al., Chin. Phys. C 36, 1603 (2012).

[37] A. Kellerbauer et al., Eur. Phys. J. D 22, 53 (2003).

[38] M. J. Oreglia, Ph.D. thesis, Stanford University, SLAC-R-236, 1980 (unpublished).

[39] A. N. Andreyev et al., Phys. Rev. C 80, 024302 (2009).

[40] R. D. Harding et al. (unpublished).

[41] T. Kibédi, T. W. Burrows, M. B. Trzhaskovskaya, P. M. Davidson, and C. W. Nestor, Nucl. Instrum. Methods Phys. Res., Sect. A 589, 202 (2008).

[42] M. Al Monthery, Ph.D. thesis, University of York, 2019 (unpublished).

[43] S. Agostinelli et al., Nucl. Instrum. Methods Phys. Res., Sect. A 506, 250 (2003).

[44] J. Allison et al., Nucl. Instrum. Methods Phys. Res., Sect. A 835, 186 (2016).

[45] E. Browne, Nucl. Data Sheets 72, 221 (1994).

[46] NNDC, Evaluated Nuclear Structure Data File, 2017.

[47] J. C. Hardy, L. C. Carraz, B. Jonson, and P. G. Hansen, Phys. Lett. B 71, 307 (1977).

[48] E. W. Otten, in Treatise on Heavy Ion Science: Volume 8: Nuclei Far From Stability, edited by D. A. Bromley (Springer, Boston, 1989), pp. 517-638.

[49] P. Campbell, I. D. Moore, and M. R. Pearson, Prog. Part. Nucl. Phys. 86, 127 (2016).

[50] M. Seliverstov et al., Hyperfine Interact. 241, 40 (2020).

[51] K. Wallmeroth, G. Bollen, A. Dohn, P. Egelhof, U. Krönert, M. J. G. Borge, J. Campos, A. Rodriguez Yunta, K. Heyde, C. De Coster, J. L. Wood, and H.-J. Kluge, Nucl. Phys. A 493, 224 (1989).

[52] P. A. Moskowitz and M. Lombardi, Phys. Lett. B 46, 334 (1973).

[53] V. J. Ehlers, Y. Kabasakal, H. A. Shugart, and O. Tezer, Phys. Rev. 176, 25 (1968).

[54] A. E. Barzakh, L. K. Batist, D. V. Fedorov, V. S. Ivanov, K. A. Mezilev, P. L. Molkanov, F. V. Moroz, S. Y. Orlov, V. N. Panteleev, and Y. M. Volkov, Phys. Rev. C 86, 014311 (2012).

[55] S. Schmidt et al., Phys. Lett. B 779, 324 (2018).

[56] U. Krönert, S. Becker, G. Bollen, M. Gerber, T. Hilberath, H. J. Kluge, and G. Passler, Z. Phys. A 331, 521 (1988).

[57] G. Savard et al., Nucl. Phys. A 512, 241 (1990).

[58] C. R. Bingham et al., Phys. Rev. C 51, 125 (1995).

[59] F. Le Blanc et al., Phys. Rev. Lett. 79, 2213 (1997).

[60] F. Ibrahim et al., Eur. Phys. J. A 10, 139 (2001). 
[61] A. Andreyev et al., CERN Report No. INTC-P-319-ADD-1, 2013 (unpublished).

[62] G. Dracoulis, B. Fabricius, R. Bark, A. Stuchbery, D. Popescu, and T. Kibedi, Nucl. Phys. A 510, 533 (1990).

[63] C. J. Gallagher and S. A. Moszkowski, Phys. Rev. 111, 1282 (1958).

[64] C. Ekström, G. Wannberg, and Y. S. Shishodia, Hyperfine Interact. 1, 437 (1975).

[65] I.-L. Lamm, Nucl. Phys. A 125, 504 (1969).

[66] R. Bengtsson, J. Dudek, W. Nazarewicz, and P. Olanders, Phys. Scr. 39, 196 (1989).

[67] M. A. Cardona et al., Phys. Rev. C 59, 1298 (1999).
[68] J. O. Rasmussen, Phys. Rev. 113, 1593 (1959).

[69] L. Ghys, A. N. Andreyev, M. Huyse, P. Van Duppen, S. Antalic, A. Barzakh, L. Capponi, T. E. Cocolios, J. Cubiss, X. Derkx, H. De Witte, J. Elseviers, F. P. Hessberger, Z. Kalaninová, U. Köster, J. F. W. Lane, V. Liberati, S. Mitsuoka, Y. Nagame, K. Nishio, S. Ota, D. Pauwels, R. D. Page, L. Popescu, D. Radulov, M. M. Rajabali, E. Rapisarda, K. Sandhu, V. L. Truesdale, P. Van den Bergh, and Y. Wakabayashi, Phys. Rev. C 100, 054310 (2019).

Correction: The sixth affiliation contained errors and has been fixed. 